\title{
A dynamic equivalence principle for systematic longevity risk management
}

\author{
HAMZA HANBALI \\ hamza.hanbali@kuleuven. be \\ JAN DHAENE \\ jan.dhaene@kuleuven.be
}

\author{
MiCHEL DENUIT \\ michel.denuit@uclouvain.be \\ JULIEN TRUFIN \\ julien.trufin@ulb.ac.be
}

February 13, 2019

\begin{abstract}
This paper addresses systematic longevity risk in long-term insurance business. We analyze the consequences of working under unknown survival probabilities on the efficiency of the Law of Large Numbers and point out the need for appropriate and feasible risk management techniques. We propose a setting for risk sharing schemes between the insurer and policyholders via a dynamic equivalence principle. We focus on a pure endowment contract and derive conditions for a viable risk sharing scheme which enhances the solvency situation of the insurer while being more favorably priced for the policyholders.
\end{abstract}

Keywords: systematic longevity risk, risk sharing, solvency, dynamic equivalence principle, (conditional) Law of Large Numbers.

\section{Introduction}

The evolution of the overall mortality pattern of a population is impacted by factors which can be either positive, due to medical advances and developments in health care, or negative, due to epidemics and other natural disasters. In the context of long-term insurance business, the fact that these factors are common to all individuals in the population induces a positive dependence between the remaining lifetimes of policyholders, implying that the independence assumption necessary for the Law of Large Numbers (LLN) is violated. Although increasing the number of identically distributed policies may help to hedge against the diversifiable part of the risk, the insurer remains exposed to a systematic part that requires alternative hedging techniques.

The solutions proposed in the existing literature to cope with the systematic risk can be summarized in two categories: internal or external hedging. Internal hedging 
essentially consists in implementing natural hedging strategies. This solution is however not sufficient, since insurance companies in practice cannot treat their business lines as financial assets; see e.g. Cox and Lin (2007) and Luciano et al. (2017) for a discussion. External hedging, on the other hand, involves a third party to which the systematic risk is transferred. The third party can for instance be a reinsurer or a pension insurer who is better able to perform the internal hedge. We refer to Blake et al. (2013) for a discussion on the pension (re)insurance market and to Denuit et al. (2011) for some limitations related to reinsurance for life insurance, the most important one being related to credit risk. The third party can also be an investor seeking for diversification opportunities. This implies that the risk is transferred in the form of a longevity-linked derivative; see Blake et al. (2013) and Blake et al. (2017), among others, for more details on the growing life market.

In this paper, we investigate how to design a product that allows to manage unpredictable longevity risk throughout the life of the contract. This is achieved by transferring this risk back to policyholders via an agreed-upon risk sharing scheme. Our contribution is threefold. First, inspired by previous work of e.g. Milevsky et al. (2006), we analyze the performance of the LLN when policyholders' future lifetimes are only conditionally independent. One of the (obvious) findings is that increasing the size of the portfolio is still beneficial for the insurer. However, the risk stemming from the uncertainty on the survival index of the portfolio cannot be eliminated, which highlights the risk of specifying the elements of the insurance contract in absolute term. Second, we propose a framework in which the information stream can be used to update the contract elements over time. Under a pricing scheme which we label the dynamic equivalence principle, the insurer and the policyholders agree upon how the experienced loss is shared between both parties on, say, a yearly basis. Third, we focus on a pure endowment contract and we provide indexing formulas for the updated premium plan and/or the benefit package. It is shown that an appropriate updating mechanism has to comply with two conditions. The first one aims at enhancing the solvency situation of the insurer. The second one follows from the fact that the premium of the contract has to be lower compared to the one from the corresponding classical contract in order to remain sufficiently appealing for policyholders. Taking into account these two constraints enables us to derive a viable updating mechanism.

Updating mechanisms shifting (part of) the burden of systematic risk back to policyholders are not new in the literature. The main idea is to design the product such that the insurer is less exposed to systematic deviations by adapting premiums, benefits and/or the date of the first benefit payment. Particularly well-known examples of such products are unit-linked policies which consist in linking the benefits to the performance of a fund, and in this way, putting the down- as well as upside systematic investment risk on the shoulders of the policyholders. Another example of products where the benefits are adapted to the experienced survival is a tontine scheme or a survival fund; see e.g. Milevsky and Salisbury (2016), Forman and Sabin (2016) as well as the tonuity proposal of Chen et al. (2017) which combines a tontine and an annuity. Contracts where systematic longevity risk is coped with by regularly updating the benefit have also been considered in Dahl (2004). Indexing may as well be related to group self-annuitization, in which 
retirees pool and form a fund to provide protection against longevity; see Piggott et al. (2005) and Valdez et al. (2006). Moreover, Denuit et al. (2011) and Richter and Frederik (2011) advocate indexing mechanisms for life annuities. We also refer to the participating variable investment-linked deferred annuities (VILDAs) studied in Mauer et al. (2013). More recently, Denuit et al. (2017) and Dhaene et al. (2017) studied premium indexing for lifelong health insurance contracts.

The present work provides a setting for updating the various contract elements. It may offer a superior protection to policyholders, since the insurer can contribute to restore the break of the actuarial equivalence. An additional advantage of working under the proposed framework is that it enables to design a risk sharing scheme which enhances the insurer's solvency. Furthermore, the proposed setting allows us to derive conditions on the yearly share and on the longevity risk loadings such that contracts priced with the dynamic equivalence principle lead to lower premiums than their classical counterparts. Note that Mauer et al. (2013) find, under a lifecycle portfolio choice model with CRRA utility function, that policyholders would be keen to purchase participating contracts provided the loading of this contract is below a certain threshold; see also Weale and van de Ven (2016). In the same spirit, Boon et al. (2018) compare the CRRA-based preferences of policyholders between annuity contracts and GSA plans and include the perspective of equity holders. Our results differ and generalize those mentioned above at different levels. The proposed setting goes beyond the no-transfer/full-transfer binarism and provides a scheme where the systematic risk is shared among the two parties. Additionally, the conditions under which each party has an advantage in engaging in the dynamic contract are given in closed-form expression. Moreover, the constraint imposed from the viewpoint of the policyholder is that the risk sharing scheme should lead to a contract where, taking into account the potential future payments, the premium has to be lower than that of the corresponding classical contract priced in a classical way. This implies that the results hold for any utility function describing the choice of a profit-seeking decision maker, and thus, no assumption is required on its functional form. Also, the loading considered here is not restricted to a given choice of the loading function and includes e.g. the quantilebased loading considered in Mauer et al. (2013) as a particular case. Last but not least, our work reconciles both the solvency constraint of the insurer and the price constraint of policyholders, leading to a viable risk sharing scheme.

The remainder of the paper is organized as follows. Section 2 addresses the systematic longevity risk and the consequences of replacing the independent remaining lifetimes assumption by a conditional independence assumption. This section is supplemented by the discussion in Appendix A. In Section 3, we introduce the dynamic equivalence principle as a possible solution to reduce the insurer's exposure to systematic risk. We apply the approach to a portfolio of pure endowments in Section 4 and show how to design a viable contract which is appealing to both the insurer and policyholders. This section contains also a numerical analysis of these viable contracts. Finally, Section 5 concludes the paper.

We end the introduction by noting that although the angle taken here is from the longevity aspect of the risk, our results and conclusions can readily be generalized to include other elements of the technical basis such as interest rates and lapse rates. 


\section{Systematic longevity risk}

Consider a portfolio of $l_{x}$ policyholders all aged $x$ at time 0 whose remaining lifetimes are denoted by $T_{1}, \ldots, T_{l_{x}}$. At time 0 , the survival-or-not upon time $t$ of policyholder $i$ is represented by the following survival indicator:

$$
I_{i}(0, t)=\left\{\begin{array}{cc}
1 & \text { if } T_{i}>t \\
0 & \text { otherwise }
\end{array},\right.
$$

whereas the corresponding survival index of the portfolio is defined as follows:

$$
I(0, t)=\frac{1}{l_{x}} \sum_{i=1}^{l_{x}} I_{i}(0, t) .
$$

Throughout the paper, as in most models for projecting mortality and survival, the conditional remaining lifetimes of the policyholders, given a particular mortality scenario $\Theta=\theta$, are assumed to be mutually independent. We introduce the notations ${ }_{t} p_{x}(\theta)$ and ${ }_{t} q_{x}(\theta)$ for the conditional survival and death probabilities, respectively:

$$
{ }_{t} p_{x}(\theta)=\mathbb{P}\left[I_{i}(0, t)=1 \mid \Theta=\theta\right]=1-{ }_{t} q_{x}(\theta) .
$$

The unconditional survival probability, which is denoted by ${ }_{t} p_{x}$, is given by:

$$
{ }_{t} p_{x}=\mathbb{P}\left[I_{i}(0, t)=1\right]=\mathbb{E}\left[{ }_{t} p_{x}(\Theta)\right],
$$

and a similar expression holds for the corresponding death probability.

In this setting, we find that the probability of joint survival for insureds $i$ and $j$, for $i \neq j$, equals

$$
\mathbb{P}\left[I_{i}(0, t)=1, I_{j}(0, t)=1\right]=\mathbb{E}\left[\left({ }_{t} p_{x}(\Theta)\right)^{2}\right] .
$$

Furthermore, from (4) and (5), we find that the covariance between the variables $I_{i}(0, t)$ and $I_{j}(0, t)$, for $i \neq j$, is given by

$$
\operatorname{Cov}\left[I_{i}(0, t), I_{j}(0, t)\right]=\operatorname{Var}\left[{ }_{t} p_{x}(\Theta)\right]
$$

which implies a non-negative dependence between the survival indicators. This expression shows that the degree of our ignorance about the future survival probability drives the dependence between the survival indicators: the more ${ }_{t} p_{x}(\Theta)$ is uncertain, in the sense that it has a larger variance, the more the survival indicators are correlated 1 . In case ${ }_{t} p_{x}(\Theta)$ is deterministic, meaning that the survival probability is known with certainty, the covariance between $I_{i}(0, t)$ and $I_{j}(0, t)$ is zero and the survival indicators are independent (since zero correlation is equivalent to independence in the Bernoulli case). This latter case is in line with the standard actuarial assumption of independence.

\footnotetext{
${ }^{1}$ We refer to Appendix $\mathrm{A}$ for a more detailed discussion on the dependence between policyholders' survival indexes and its consequence on the LLN.
} 
Using the law of total variance, we find that $\operatorname{Var}[I(0, t)]$ can be split into two parts:

$$
\operatorname{Var}[I(0, t)]=\frac{1}{l_{x}} \mathbb{E}\left[{ }_{t} p_{x}(\Theta){ }_{t} q_{x}(\Theta)\right]+\operatorname{Var}\left[{ }_{t} p_{x}(\Theta)\right]
$$

The diversifiable part of the insurance risk is captured by the first term in (7), which depends on the size of the portfolio. The second term is related to the uncertainty on the survival probabilities, and hence, captures the systematic part. This two-components representation implies that the variance of the average benefit payment is a decreasing function of the number of insureds. The cases where $l_{x}$ goes to infinity and to 1 lead to the following lower and upper bounds:

$$
\operatorname{Var}\left[{ }_{t} p_{x}(\Theta)\right] \leq \operatorname{Var}[I(0, t)] \leq \operatorname{Var}\left[I_{i}(0, t)\right]
$$

implying that it is beneficial to increase the size of the portfolio; see Appendix A for further discussion.

The main conclusion of this section is that in case of conditional independence, only part of the insurance risk can be diversified by pooling. Taking into account this observation, it seems that specifying the elements of the insurance contract in absolute terms at policy issue can be extremely dangerous, because of the substantial systematic longevity risk captured in $\operatorname{Var}\left[{ }_{t} p_{x}(\Theta)\right]$.

\section{Dynamic equivalence principle}

\subsection{The actuarial equivalence principle at contract initiation}

The main idea behind the proposed risk sharing scheme is that at the end of each period, the retrospective reserve is compared to the required liabilities (determined prospectively, taking into account new information on the insured risks). The experienced loss, that is the difference between the retrospective and prospective reserves, is then shared between the policyholder and the insurer, according to an agreed-upon risk sharing scheme which is determined at policy issue. We focus in this section on deriving rules for a risk sharing scheme for insurance contracts with survival benefits but the approach consisting in sharing the experienced loss is applicable to other types of insurance contracts, including those with a death benefit.

We consider an insurance contract sold to $l_{x}$ policyholders aged $x$ at time 0 . The policy stipulates that upon survival at times $k+1, k=0,1,2, \ldots$, the beneficiary will receive the amount $b_{k+1}^{(0)} \geq 0$, while upon survival at time $k$, the policyholder will pay the

premium $\pi_{k}^{(0)} \geq 0$. Given the information available at time 0 , hence, the superscript ${ }^{(0)}$, we denote by $\mathcal{C}_{0}^{(0)}$ the contract elements agreed-upon at time 0 :

$$
\mathcal{C}_{0}^{(0)}=\left\{\underline{b}_{0}^{(0)}, \underline{\pi}_{0}^{(0)}\right\},
$$


where $\underline{b}_{0}^{(0)}=\left(b_{1}^{(0)}, b_{2}^{(0)}, \ldots\right)$ and $\underline{\pi}_{0}^{(0)}=\left(\pi_{0}^{(0)}, \pi_{1}^{(0)}, \ldots\right)$ are the benefit package and the premium plan, respectively. The present value at time 0 of all future benefit payments per-policy is denoted by the random variable $B_{0}^{(0)}$ :

$$
B_{0}^{(0)}=\sum_{j=0}^{\infty} b_{j+1}^{(0)} v^{j+1}{ }_{j+1} p_{x}(\Theta),
$$

whereas the present value of all future premium payments is given by the random variable $\Pi_{0}^{(0)}$ :

$$
\Pi_{0}^{(0)}=\sum_{j=0}^{\infty} \pi_{j}^{(0)} v^{j}{ }_{j} p_{x}(\Theta),
$$

where $v$ is the constant yearly discounting factor. The loss random variable at time 0 is denoted by $L_{0}$ and is defined as the present value of future benefits minus future premiums, i.e.:

$$
L_{0}=B_{0}^{(0)}-\Pi_{0}^{(0)}
$$

Recall that the $j$-year survival of policyholder $i$ is characterized by the conditional probability ${ }_{j} p_{x}(\Theta)$. Based on the knowledge available at time 0 and by using the notation $\mathcal{F}_{0}$, the actuary attaches the following values to these probabilities:

$$
\mathbb{E}\left[{ }_{j} p_{x}(\Theta) \mid \mathcal{F}_{0}\right]={ }_{j} p_{x}^{(0)},
$$

for $j=1,2, \ldots$. Henceforth, we will also use the superscript ${ }^{(k)}$ to indicate quantities that are based on information and expert opinion available at time $k, k=1,2, \ldots$, and we replace the notation $\mathbb{E}\left[. \mid \mathcal{F}_{k}\right]$ by $\mathbb{E}_{k}[$.$] . Note that apart from the probabilities introduced$ above, at time 0 , the actuary also has to determine other factors of the technical basis.

The expected present value of the benefit and premium cash flow streams are then given by the following expected present values:

$$
\mathbb{E}_{0}\left[B_{0}^{(0)}\right]=\sum_{j=0}^{\infty} b_{j+1}^{(0)} v^{j+1}{ }_{j+1} p_{x}^{(0)},
$$

and

$$
\mathbb{E}_{0}\left[\Pi_{0}^{(0)}\right]=\sum_{j=0}^{\infty} \pi_{j}^{(0)} v^{j}{ }_{j} p_{x}^{(0)},
$$

respectively. We assume that the premiums and benefits of the contract are set such that they fulfill the actuarial equivalence principle, i.e. such that the expected value of the loss in (9), conditionally on the information available at time 0 , is equal to 0 at that time:

$$
\mathbb{E}_{0}\left[L_{0}\right]=0 \Longleftrightarrow \mathbb{E}_{0}\left[B_{0}^{(0)}\right]=\mathbb{E}_{0}\left[\Pi_{0}^{(0)}\right]
$$

The valuation approach based on the actuarial equivalence principle (11) is the one commonly used in practice for classical life insurance, where the benefit and premium cash 
flow streams $\underline{b}_{0}^{(0)}$ and $\underline{\pi}_{0}^{(0)}$ are fixed at policy issue and remain, in principle, unchanged during the life of the contract. This means that the risk of having chosen a wrong technical basis is fully taken by the insurer. In order to cope with this risk, the insurer commonly charges a (implicit) loading which is used to cover the diversifiable as well as the undiversifiable parts of the risk. In this section, we discard this loading, but we will include it in Section 4 .

\subsection{The dynamic equivalence principle}

Let us now consider a non-classical life insurance contract whose features can be changed over time. In contrast to classical insurance pricing, we allow to take into account the new knowledge about the survival probabilities.

Suppose that we have arrived at time 1 and that the policy is still in force. Assuming that the technical interest rate is guaranteed, the available provision (or reserve) at that time is given by:

$$
V_{1}^{(0)}=\frac{\pi_{0}^{(0)}}{v I(0,1)},
$$

where $I(0,1)$ is the observed survival index over the first year which is assumed to be strictly greater than 0 . This assumption means that there is at least one survivor at time 1. In case $I(0,1)=0$ then all contracts are terminated at time 1 and no updating is needed anymore. Note that throughout the paper and for any $k=1,2, \ldots$, the reserves are calculated before benefit and premium payment.

Having arrived at time 1 , the realization $I(0,1)$ provides the insurer with additional information. Moreover, assuming that the insured $i$ is still alive, we know that $I_{i}(0,1)=1$. Based on this and other knowledge $\mathcal{F}_{1}$ available at time 1 , we obtain the following updated survival probabilities:

$$
\mathbb{E}_{1}\left[{ }_{j} p_{x+1}(\Theta)\right]={ }_{j} p_{x+1}^{(1)}, \quad j=1,2, \ldots
$$

From (12), it follows that the required reserve based on the new information is given by:

$$
\mathbb{E}_{1}\left[B_{1}^{(0)}-\Pi_{1}^{(0)}\right]=\sum_{j=0}^{\infty} b_{j+1}^{(0)} v^{j}{ }_{j} p_{x+1}^{(1)}-\sum_{j=0}^{\infty} \pi_{j+1}^{(0)} v^{j}{ }_{j} p_{x+1}^{(1)} .
$$

In general, the actuarial equivalence will be broken because the realization of the retrospective reserve deviates from its assumption and the estimate of the technical basis has changed. In order to restore it, the following capital is required:

$$
\mathbb{E}_{1}\left[B_{1}^{(0)}-\Pi_{1}^{(0)}\right]-V_{1}^{(0)}
$$

which represents the deviation between the required and the retrospective reserve at time 1. We suppose now that this required amount is shared among the insurer and the policyholder. For $\alpha_{1} \in[0,1]$, let $1-\alpha_{1}$ be the share of the loss covered by the insurer 
whereas the contribution of the policyholder is given by $\alpha_{1}\left(\mathbb{E}_{1}\left[B_{1}^{(0)}-\Pi_{1}^{(0)}\right]-V_{1}^{(0)}\right)$. On the one hand, the retrospective reserve after having been increased by the insurer's participation is given by:

$$
V_{1}^{(1)}=V_{1}^{(0)}+\left(1-\alpha_{1}\right)\left(\mathbb{E}_{1}\left[B_{1}^{(0)}-\Pi_{1}^{(0)}\right]-V_{1}^{(0)}\right)
$$

On the other hand, from time 1 on, the remaining premium plan $\underline{\pi}_{1}^{(0)}$ is replaced by $\underline{\pi}_{1}^{(1)}$ :

$$
\underline{\pi}_{1}^{(1)}=\left(\pi_{1}^{(1)}, \pi_{2}^{(1)}, \ldots\right)
$$

and the remaining benefit package $\underline{b}_{1}^{(0)}$ is replaced by $\underline{b}_{1}^{(1)}$ :

$$
\underline{b}_{1}^{(1)}=\left(b_{1}^{(1)}, b_{2}^{(1)}, \ldots\right) \text {. }
$$

We denote the expected actuarial present value at time 1 of the updated remaining premiums and benefits as:

$$
\mathbb{E}_{1}\left[\Pi_{1}^{(1)}\right]=\sum_{j=0}^{\infty} \pi_{j+1}^{(1)} v^{j}{ }_{j} p_{x+1}^{(1)}
$$

and

$$
\mathbb{E}_{1}\left[B_{1}^{(1)}\right]=\sum_{j=0}^{\infty} b_{j+1}^{(1)} v^{j}{ }_{j} p_{x+1}^{(1)},
$$

respectively. The updated premium plan and benefit package are determined such that the actuarial equivalence is restored at time 1, i.e. when the following equation is satisfied:

$$
\mathbb{E}_{1}\left[\Pi_{1}^{(1)}-B_{1}^{(1)}\right]=\mathbb{E}_{1}\left[\Pi_{1}^{(0)}-B_{1}^{(0)}\right]+\alpha_{1}\left(\mathbb{E}_{1}\left[B_{1}^{(0)}-\Pi_{1}^{(0)}\right]-V_{1}^{(0)}\right)
$$

Taking into account the contributions of both the insurer and the policyholders, one finds that:

$$
V_{1}^{(1)}=\mathbb{E}_{1}\left[B_{1}^{(1)}-\Pi_{1}^{(1)}\right]
$$

which means that the actuarial equivalence has been restored at time 1 .

A similar reasoning can be applied at time 2 where the available reserve based on the updating up to time 1 is given by:

$$
V_{2}^{(1)}=\frac{V_{1}^{(1)}+\pi_{1}^{(1)}-b_{1}^{(1)}}{v I(1,2)}
$$

with $I(1,2)$ being the survival index of the portfolio from time 1 to time 2 , and assumed to be strictly positive. Using the new information available at time 2 , the estimates of the future survival probabilities at that time are updated to ${ }_{j} p_{x+2}^{(2)}$, for $j=1,2, \ldots$, such that the value of the required reserve is as follows:

$$
\mathbb{E}_{2}\left[B_{2}^{(1)}-\Pi_{2}^{(1)}\right]=\sum_{j=0}^{\infty} b_{j+2}^{(1)} v^{j}{ }_{j} p_{x+2}^{(2)}-\sum_{j=0}^{\infty} \pi_{j+2}^{(1)} v_{j}{ }_{j} p_{x+2}^{(2)} .
$$


The amount needed to restore the actuarial equivalence is $\mathbb{E}_{2}\left[B_{2}^{(1)}-\Pi_{2}^{(1)}\right]-V_{2}^{(1)}$. For $\alpha_{2} \in[0,1]$, the share of the insurer is given by $1-\alpha_{2}$, such that:

$$
V_{2}^{(2)}=V_{2}^{(1)}+\left(1-\alpha_{2}\right)\left(\mathbb{E}_{2}\left[B_{2}^{(1)}-\Pi_{2}^{(1)}\right]-V_{2}^{(1)}\right)
$$

The contribution of the policyholder is $\alpha_{2}\left(\mathbb{E}_{2}\left[B_{2}^{(1)}-\Pi_{2}^{(1)}\right]-V_{2}^{(1)}\right)$. The updated premium plan $\underline{\pi}_{2}^{(2)}$ and benefit package $\underline{b}_{2}^{(2)}$ are determined from the following updated actuarial equivalence:

$$
\mathbb{E}_{2}\left[\Pi_{2}^{(2)}-B_{2}^{(2)}\right]=\mathbb{E}_{2}\left[\Pi_{2}^{(1)}-B_{2}^{(1)}\right]+\alpha_{2}\left(\mathbb{E}_{2}\left[B_{2}^{(1)}-\Pi_{2}^{(1)}\right]-V_{2}^{(1)}\right)
$$

Insurance regulation requires that having arrived at time $k$, the actuarial equivalence has to be restored. In case of a classical life insurance contract, where benefits and premiums are fixed at policy issue, the insurer is fully responsible for restoring the actuarial equivalence, i.e. $\alpha_{k}=0$. In our present setting, the cost of restoring the actuarial equivalence is covered by both the insurer and policyholders. The available provision as well as all future benefits and premiums are updated according to a pre-specified risk sharing scheme which is characterized by the coefficients $\alpha_{1}, \alpha_{2}, \ldots$. This flexible approach to manage longevity risk for newly underwritten contracts can be see as a series of successive (yearly) applications of the fundamental static equivalence principle. We say here that such a contract is managed by a dynamic equivalence principle, which is defined hereafter for any time $k$.

Definition 1 (Dynamic equivalence principle) At any time $k$, the $k-1$ values of the remaining contract features

$$
\mathcal{C}_{k}^{(k-1)}=\left\{\underline{b}_{k}^{(k-1)}, \underline{\pi}_{k}^{(k-1)}\right\}
$$

are replaced by

$$
\mathcal{C}_{k}^{(k)}=\left\{\underline{b}_{k}^{(k)}, \underline{\pi}_{k}^{(k)}\right\}
$$

taking into account the information stream over time, such that the actuarial equivalence is restored at that time, i.e.

$$
\mathbb{E}_{k}\left[\Pi_{k}^{(k)}-B_{k}^{(k)}\right]=\mathbb{E}_{k}\left[\Pi_{k}^{(k-1)}-B_{k}^{(k-1)}\right]+\alpha_{k}\left(\mathbb{E}_{k}\left[B_{k}^{(k-1)}-\Pi_{k}^{(k-1)}\right]-V_{k}^{(k-1)}\right),
$$

where $\alpha_{k} \in[0,1]$ is the share of the loss born by the policyholder at time $k$. The retrospective reserve at time $k$ before updating, i.e. $V_{k}^{(k-1)}$, is given by:

$$
V_{k}^{(k-1)}=\frac{V_{k-1}^{(k-1)}+\pi_{k-1}^{(k-1)}-b_{k-1}^{(k-1)}}{v I(k-1, k)},
$$


where $V_{k-1}^{(k-1)}$ is the retrospective reserve at time $k-1$ after having been increased by the insurer's participation in the deviation risk:

$$
V_{k-1}^{(k-1)}=V_{k-1}^{(k-2)}+\left(1-\alpha_{k-1}\right)\left(\mathbb{E}_{k-1}\left[B_{k-1}^{(k-2)}-\Pi_{k-1}^{(k-2)}\right]-V_{k-1}^{(k-2)}\right),
$$

and where $I(k-1, k)$ represents the (strictly positive) survival index from time $k-1$ to time $k$ in the portfolio which is composed of policyholders aged $x+k-1$ at time $k-1$. Taking into account the contribution of both the insurer and the policyholders, one finds that:

$$
V_{k}^{(k)}=\mathbb{E}_{k}\left[B_{k}^{(k)}-\Pi_{k}^{(k)}\right],
$$

which means that the actuarial equivalence has been restored at time $k$.

\section{A pure endowment contract with single premium}

\subsection{The updating mechanism}

In this section, we focus on a portfolio of $t$-year pure endowments sold to $l_{x}$ policyholders aged $x$ at time 0 . The contract pays a benefit of $b_{t}^{(0)}$ at time $t$ upon survival of the policyholder at that time, and is sold for a single pure premium $\pi_{0}^{(0)}$. We assume that the policy is flexible, in the sense that it allows the contract elements to be updated over time. Based on the estimate ${ }_{t} p_{x}^{(0)}$ at time 0 of the $t$-year survival probability, we use the actuarial equivalence principle 10 to determine the value of the pure premium $\pi_{0}^{(0)}$ at contract inception:

$$
\pi_{0}^{(0)}=b_{t}^{(0)} v^{t}{ }_{t} p_{x}^{(0)} .
$$

We include a premium loading in this section and we denote it by $\varphi$, which is assumed to be positive. This results in the loaded single premium $P_{0}^{(0)}$ :

$$
P_{0}^{(0)}=\pi_{0}^{(0)}+\varphi
$$

This setting includes a wide range of possible pricing principles; see Kaas et al. (2008) for some examples. The loading $\varphi$ can also be determined by applying shocks on the estimated survival probabilities, in the spirit of the Solvency II regulation. Because the loaded premium is the sum of the actuarial value of the benefit $b_{t}^{(0)}$ and a loading, we can interpret $P_{0}^{(0)}$ as the pure premium for a contract paying the benefit $b_{t}^{(0)}+\frac{\varphi}{v^{t}{ }_{t} p_{x}^{(0)}}$ at maturity. Thus, the contract can be described by the benefit package $\underline{b}_{0}^{(0)}=\left(0, \ldots, 0, b_{t}^{(0)}+\right.$ $\left.\frac{\varphi}{v^{t}{ }_{t} p_{x}^{(0)}}, 0, \ldots\right)$ and the pure premium plan $\underline{P}_{0}^{(0)}=\left(P_{0}^{(0)}, 0,0, \ldots\right)$. Under this setting, the insurer estimates at time 0 both the pure premium and the loading. As the future unfolds and new information becomes available, the estimate of the premium may require an adjustment and the estimate of the loading may turn out to be insufficient to cope with the deviation risk for the remaining years. In the sequel, the time- 0 estimates of the pure premium as well as the loading will both be updated over time. Note that another simpler setting which is not considered here and requires only minor modifications in the 
subsequent results is to update the pure premium only, without taking into account the loading in the dynamic equivalence principle.

At time 1, the value of the retrospective reserve per policy still in force is:

$$
V_{1}^{(0)}=\frac{P_{0}^{(0)}}{v I(0,1)}
$$

The new information available at time 1 leads to a new estimate ${ }_{t-1} p_{x+1}^{(1)}$ of the $(t-1)$ year survival probability of the policyholder now aged $x+1$. The required reserve is then estimated at time 1 by $b_{t}^{(0)} v_{t-1}^{t-1} p_{x+1}^{(1)}$.

From time 1 on, the benefit package $\underline{b}_{1}^{(0)}=\left(0, \ldots, 0, b_{t}^{(0)}+\frac{\varphi}{v^{t}{ }_{t} p_{x}^{(0)}}, 0, \ldots\right)$ and the premium plan $\underline{P}_{1}^{(0)}=(0,0,0, \ldots)$ at that time are replaced by $\underline{b}_{1}^{(1)}=\left(0, \ldots, 0, b_{t}^{(1)}+\frac{\varphi}{v^{t}{ }_{t} p_{x}^{(0)}}, 0, \ldots\right)$ and $\underline{P}_{1}^{(1)}=\left(P_{1}^{(1)}, 0,0, \ldots\right)$, respectively, where we assume that only a single extra-premium $P_{1}^{(1)}$ is paid at time 1 . This means that the benefit is updated from $b_{t}^{(0)}$ to $b_{t}^{(1)}$, and/or an additional amount $P_{1}^{(1)}$ is paid by the policyholders.

Let us now apply the dynamic equivalence principle (17) to obtain the values of the updated benefit and the additional premium. The present values of future premiums using the information available at time 1 before and after the updating are given by:

$$
\mathbb{E}_{1}\left[\Pi_{1}^{(1)}\right]=P_{1}^{(1)}
$$

and

$$
\mathbb{E}_{1}\left[\Pi_{1}^{(0)}\right]=0
$$

respectively. Moreover, the present values of future benefits using the information available at time 1 before and after the updating are given by:

$$
\mathbb{E}_{1}\left[B_{1}^{(1)}\right]=\left(b_{t}^{(1)}+\frac{\varphi}{v^{t}{ }_{t} p_{x}^{(0)}}\right) v^{t-1}{ }_{t-1} p_{x+1}^{(1)},
$$

and

$$
\mathbb{E}_{1}\left[B_{1}^{(0)}\right]=\left(b_{t}^{(0)}+\frac{\varphi}{v^{t}{ }_{t} p_{x}^{(0)}}\right) v^{t-1}{ }_{t-1} p_{x+1}^{(1)},
$$

respectively. Plugging Expressions $(20)-(23)$ as well as $(19)$ in Equation (17) and multiplying both sides of the resulting equation by $\left(-v_{t-1}^{t-1} p_{x+1}^{(1)}\right)^{-1}$ leads to:

$$
b_{t}^{(1)}-\frac{P_{1}^{(1)}}{v^{t-1}{ }_{t-1} p_{x+1}^{(1)}}=b_{t}^{(0)}-\alpha_{1}\left(\left(b_{t}^{(0)}+\frac{\varphi}{v^{t}{ }_{t} p_{x}^{(0)}}\right)-\frac{P_{0}^{(0)}}{v^{t} I(0,1)_{t-1} p_{x+1}^{(1)}}\right) .
$$

Furthermore, by noting that $P_{0}^{(0)}=\left(b_{t}^{(0)}+\frac{\varphi}{v^{t}{ }_{t} p_{x}^{(0)}}\right) v^{t}{ }_{t} p_{x}^{(0)}$, we find that $b_{t}^{(1)}$ and $P_{1}^{(1)}$ satisfy the following equation:

$$
b_{t}^{(1)}-\frac{P_{1}^{(1)}}{v^{t-1}{ }_{t-1} p_{x+1}^{(1)}}=b_{t}^{(0)}-\alpha_{1}\left(b_{t}^{(0)}+\frac{\varphi}{v^{t}{ }_{t} p_{x}^{(0)}}\right)\left(1-\frac{{ }_{t} p_{x}^{(0)}}{I(0,1)_{t-1} p_{x+1}^{(1)}}\right) .
$$


Using (25), we find that the retrospective reserve at time 2 is given by:

$$
V_{2}^{(1)}=\left(b_{t}^{(1)}+\frac{\varphi}{v^{t}{ }_{t} p_{x}^{(0)}}\right) v^{t-2} \frac{{ }_{t-1} p_{x+1}^{(1)}}{I(1,2)} .
$$

If we apply the dynamic equivalence principle (17), we find that the time-2 updated value of the benefit $b_{t}^{(2)}$ and the additional premium $P_{2}^{(2)}$ satisfy the equation:

$$
b_{t}^{(2)}-\frac{P_{2}^{(2)}}{v^{t-2}{ }_{t-2} p_{x+2}^{(2)}}=b_{t}^{(1)}-\alpha_{2}\left(b_{t}^{(1)}+\frac{\varphi}{v^{t}{ }_{t} p_{x}^{(0)}}\right)\left(1-\frac{{ }_{t-1} p_{x+1}^{(1)}}{I(1,2)_{t-2} p_{x+2}^{(2)}}\right) .
$$

In general, the retrospective reserve $V_{k}^{(k-1)}$ at time $k$, for $k=2, \ldots, t$, is given by:

$$
V_{k}^{(k-1)}=\left(b_{t}^{(k-1)}+\frac{\varphi}{v^{t}{ }_{t} p_{x}^{(0)}}\right) v^{t-k} \frac{t_{-k+1} p_{x+k-1}^{(k-1)}}{I(k-1, k)},
$$

such that $b_{t}^{(k)}$ and $P_{k}^{(k)}$ satisfy:

$$
b_{t}^{(k)}-\frac{P_{k}^{(k)}}{v^{t-k}{ }_{t-k} p_{x+k}^{(k)}}=b_{t}^{(k-1)}-\alpha_{k}\left(b_{t}^{(k-1)}+\frac{\varphi}{v^{t}{ }_{t} p_{x}^{(0)}}\right)\left(1-\frac{{ }_{t-k+1} p_{x+k-1}^{(k-1)}}{I(k-1, k)_{t-k} p_{x+k}^{(k)}}\right) \text {. }
$$

Thus, the values of the couple $\left(b_{t}^{(k)}, P_{k}^{(k)}\right)$ depend on the realization of the survival index, the new estimate of the mortality table and the participation of the insurer to cover the systematic deviation through the choice of the parameters $\alpha_{1}, \ldots, \alpha_{k}$. However, there is no unique couple which can be determined from (26). Hereafter, we consider two particular cases; updating the premium plan only, and updating the benefit package only.

Case 1 (Updating the premium plan only) In case the benefit is not subject to revision, we find that the reserve at time $k$ is as follows:

$$
V_{k}^{(k-1)}=\frac{\left(b_{t}^{(0)}+\frac{\varphi}{v^{t}{ }_{t} p_{x}^{(0)}}\right) v^{t-k+1}{ }_{t-k+1} p_{x+k-1}^{(k-1)}}{v I(k-1, k)}=\frac{P_{0}^{(0)}}{v^{k}{ }_{t} p_{x}^{(0)}} \frac{t-k+1}{I(k-1, k)} p_{x+k-1}^{(k-1)} .
$$

Let us now derive a general expression for the additional premium. Starting with the time-1 additional amount $P_{1}^{(1)}$, we find from (25):

$$
P_{1}^{(1)}=\alpha_{1}\left(\left(b_{t}^{(0)}+\frac{\varphi}{v^{t}{ }_{t} p_{x}^{(0)}}\right) v^{t-1}{ }_{t-1} p_{x+1}^{(1)}-\frac{P_{0}^{(0)}}{v I(0,1)}\right)=\alpha_{1} \frac{P_{0}^{(0)}}{v_{t} p_{x}^{(0)}}\left({ }_{t-1} p_{x+1}^{(1)}-\frac{{ }_{t} p_{x}^{(0)}}{I(0,1)}\right) .
$$

The premium $P_{1}^{(1)}$ corresponds to the value at time 1 of the loaded single premium using the time-0 information. This is then corrected from the deviation $\left({ }_{t-1} p_{x+1}^{(1)}-\frac{{ }_{t} p_{x}^{(0)}}{I(0,1)}\right)$ and scaled by the contribution $\alpha_{1}$ of the policyholders. Thus, the contribution of the policyholders 
takes into account changes of both the past realizations and the new estimates. At time 2, the additional amount $P_{2}^{(2)}$ is given by:

$$
P_{2}^{(2)}=\alpha_{2}\left(\left(b_{t}^{(0)}+\frac{\varphi}{v^{t}{ }_{t} p_{x}^{(0)}}\right) v^{t-2}{ }_{t-2} p_{x+2}^{(2)}-V_{2}^{(1)}\right)=\alpha_{2} \frac{P_{0}^{(0)}}{v^{2}{ }_{t} p_{x}^{(0)}}\left({ }_{t-2} p_{x+2}^{(2)}-\frac{{ }_{t-1} p_{x+1}^{(1)}}{I(1,2)}\right),
$$

and has a similar interpretation as the time-1 additional amount.

In general, we find that the additional amounts $P_{k}^{(k)}$ required from the policyholders at the successive times $k=2,3, \ldots, t$ are given by:

$$
P_{k}^{(k)}=\alpha_{k} \frac{P_{0}^{(0)}}{v^{k}{ }_{t} p_{x}^{(0)}}\left({ }_{t-k} p_{x+k}^{(k)}-\frac{t-k+1}{I(k-1, k)} p_{x+k-1}^{(k-1)}\right) .
$$

The numerical value of $P_{k}^{(k)}$ can be negative, implying that the insurer pays back the policyholder for being too conservative. Also, by considering that $k$ takes values $1,2, \ldots, t$, we implicitly assume that policyholders could pay an additional positive amount $P_{t}^{(t)}$ at contract expiration. This assumption may not be realistic in practice. However, in such a case, this can be compensated by a benefit reduction.

Let us notice that in case we use the multiplicative form $P_{0}^{(0)}=\pi_{0}^{(0)}(1+\varphi)$ instead of the additive form $P_{0}^{(0)}=\pi_{0}^{(0)}+\varphi$, the updating formula 29) implies that

$$
P_{k}^{(k)}=\pi_{k}^{(k)}(1+\varphi),
$$

where $\pi_{k}^{(k)}$ is the time-k pure additional amount which satisfies 29], such that:

$$
\pi_{k}^{(k)}=\alpha_{k} \frac{\pi_{0}^{(0)}}{v^{k}{ }_{t} p_{x}^{(0)}}\left({ }_{t-k} p_{x+k}^{(k)}-\frac{t-k+1}{I(k-1, k)} p_{x+k-1}^{(k-1)}\right) .
$$

This means that the loading $\varphi$ is constant over time and is applied to the future pure additional amounts, even when they are negative. However, we can still write the future required amounts in the additive form

$$
P_{k}^{(k)}=\pi_{k}^{(k)}+\varphi^{(k)},
$$

where $\varphi^{(k)}=\frac{\varphi}{\pi_{k}^{(k)}}$ is a time-varying loading.

Case 2 (Updating the benefit package only) Let us now assume that policyholders do not pay additional premiums. Instead, the benefit at time $t$ can be revised throughout the contract to account for deviations. In this case, it follows directly from (26) that the time- $k$ updated value of the benefit has the following expression:

$$
b_{t}^{(k)}=b_{t}^{(k-1)}-\alpha_{k}\left(b_{t}^{(k-1)}+\frac{\varphi}{v^{t}{ }_{t} p_{x}^{(0)}}\right)\left(1-\frac{p_{x+k-1}^{(k-1)}}{I(k-1, k)} \frac{{ }_{t-k} p_{x+k}^{(k-1)}}{{ }_{t-k} p_{x+k}^{(k)}}\right) .
$$

Note that we also find the following expression for the reserve at time $k$ :

$$
V_{k}^{(k-1)}=\frac{\left(1-\alpha_{k-1}\right)\left(b_{t}^{(k-1)}+\frac{\varphi}{v^{t}{ }_{t}^{(0)}}\right) v^{t-k}{ }_{t-k+1} p_{x+k-1}^{(k-1)}+\alpha_{k-1} V_{k}^{(k-1)}}{v I(k-1, k)} .
$$


Cases 1 and 2 are two particular risk sharing schemes which have the same goal of restoring the actuarial equivalence. In the remainder of the paper, we focus on Case 1 and set $b_{t}^{(t)}=b_{t}^{(0)}=b_{t}$. Moreover, we simplify the setting by assuming that $\alpha_{k}=\alpha$ for all $k=1,2, \ldots, t$, meaning that the insurer transfers back to the policyholders the same share of the shortfall every year. We answer two specific questions. The first question is raised by the insurer who wants to determine under what condition a contract managed by the dynamic equivalence principle provides more safety than a classical one, where safety is measured by the probability of loss. Second, from the point of view of policyholders, we search for conditions under which buying a contract priced under the dynamic equivalence principle will be cheaper than its classical counterpart. Here, a classical pure endowment contract is a contract without updating (i.e. $\alpha=0$ ) whose single premium is denoted by $P_{\Psi}$ :

$$
P_{\Psi}=\pi_{0}^{(0)}+\Psi
$$

with $\Psi>0$. Note that despite not being formally imposed here, it is reasonable to expect that $\varphi \leq \Psi$. Moreover, the contracts should have the same loading when the insurer covers all the shortfall, i.e. $\varphi=\Psi$ for $\alpha=0$, whereas there should be no loading when the policyholders are covering the shortfall, i.e. $\varphi=0$ for $\alpha=1$.

\subsection{Impact on the insurer's solvency}

The analysis in this subsection is carried out from the point of view of the insurer who has the choice between selling a pure endowment under the classical setting or under the risk sharing scheme described in Case 1. The time-0 shortfall risk per-policy for a portfolio of pure endowments priced in the classical static setting is denoted by $R_{\Psi}$, whereas its counterpart priced in the dynamic setting is denoted by $R_{\alpha, \varphi}$. For the classical contract, we have:

$$
R_{\Psi}=I(0, t) b_{t} v^{t}-P_{\Psi}
$$

which can also be written as:

$$
R_{\Psi}=\left(\frac{I(0, t)}{{ }_{t} p_{x}^{(0)}}-1\right) \pi_{0}^{(0)}-\Psi .
$$

On the other hand, we find in the dynamic case:

$$
R_{\alpha, \varphi}=I(0, t) b_{t} v^{t}-P_{0}^{(0)}-\sum_{k=1}^{t} v^{k} I(0, k) P_{k}^{(k)},
$$

From (29), we see that $R_{\alpha, \varphi}$ can also be written as:

$$
R_{\alpha, \varphi}=I(0, t) b_{t}^{(0)} v^{t}-P_{0}^{(0)}-\alpha \frac{P_{0}^{(0)}}{{ }_{t} p_{x}^{(0)}} \sum_{k=1}^{t} I(0, k)\left({ }_{t-k} p_{x+k}^{(k)}-\frac{{ }_{t-k+1} p_{x+k-1}^{(k-1)}}{I(k-1, k)}\right),
$$

or, in its simplified form:

$$
R_{\alpha, \varphi}=\left(\frac{I(0, t)}{{ }_{t} p_{x}^{(0)}}-1\right)\left(\pi_{0}^{(0)}-\alpha P_{0}^{(0)}\right)-\varphi
$$


where we use

$$
\sum_{k=1}^{t} I(0, k)\left({ }_{t-k} p_{x+k}^{(k)}-\frac{t-k+1}{I(k-1, k)} p_{x+k-1}^{(k-1)}\right)=I(0, t)-{ }_{t} p_{x}^{(0)}
$$

The insurer will have a loss in case the shortfall is positive, i.e. in case the payments to the policyholders are higher than expected. In the following theorem, we provide a condition on $\Psi, \varphi$ and $\alpha$ such that the probability of a loss for a contract priced under the dynamic equivalence principle is lower compared to its classical counterpart.

\section{Theorem 1 If}

$$
\mathbb{P}\left[R_{\alpha, \varphi} \geq 0\right]<\mathbb{P}\left[R_{\Psi} \geq 0\right]
$$

then $\alpha, \varphi$ and $\Psi$ have to satisfy:

$$
\frac{\pi_{0}^{(0)}}{\pi_{0}^{(0)}+\varphi}\left(1-\frac{\varphi}{\Psi}\right) \leq \alpha<\frac{\pi_{0}^{(0)}}{\pi_{0}^{(0)}+\varphi}
$$

Proof. From $(33)$ and (34), we can write $(35)$ as follows:

$$
\mathbb{P}\left[\frac{I(0, t)}{{ }_{t} p_{x}^{(0)}}-1 \geq \frac{\varphi}{\pi_{0}^{(0)}-\alpha P_{0}^{(0)}}\right]<\mathbb{P}\left[\frac{I(0, t)}{{ }_{t} p_{x}^{(0)}}-1 \geq \frac{\Psi}{\pi_{0}^{(0)}}\right],
$$

which implies that

$$
\frac{\varphi}{\pi_{0}^{(0)}-\alpha P_{0}^{(0)}} \geq \frac{\Psi}{\pi_{0}^{(0)}} .
$$

A necessary condition in order for (37) to be satisfied is given by

$$
\pi_{0}^{(0)}>\alpha P_{0}^{(0)}
$$

and provides the upper bound in (36). Moreover, rearranging (37) leads to the corresponding lower bound.

Theorem 1 shows that in case the insurer wants to reduce the loss probability, the proportion $\alpha$ of the risk that is borne by the policyholders has to be set according to (36). This observation raises the question of whether policyholders would be interested in buying such contracts. We investigate this question in the following subsection.

\subsection{Policyholders' perspective}

The goal of this subsection is to derive an additional constraint on the loadings and on the risk sharing scheme. In particular, we take into account the constraint that contracts

priced under the dynamic equivalence principle should have lower premiums compared to their classical counterparts. 
Let us first derive the actuarial value of the premiums per-policy for each contract. Obviously, for the classical contract we simply have $P_{\Psi}$. For the dynamic contract, policyholders pay the single premium $P_{0}^{(0)}$ at time 0 and potentially some additional amounts $P_{k}^{(k)}$. The time-0 random present value of all payments per-policy is given by

$$
\Pi=P_{0}^{(0)}+\sum_{k=1}^{t} I(0, k) P_{k}^{(k)} v^{k}=P_{0}^{(0)}+\alpha \frac{P_{0}^{(0)}}{{ }_{t} p_{x}^{(0)}}\left(I(0, t)-{ }_{t} p_{x}^{(0)}\right) .
$$

We find immediately that $\mathbb{E}_{0}[\Pi]=P_{0}^{(0)}$, which implies that policyholders are not expected to pay additional premiums.

We now state the theorem showing that under a condition on $\Psi, \varphi$ and $\alpha$, it remains favorable to buy a contract under the dynamic setting, although policyholders may have to pay additional amounts in the future. The reasoning here is based on the fact that if the dynamic contract leads to lower premiums (including the future potential payments), then it would be preferred by profit-seeking policyholders regardless of their risk preference.

Theorem 2 A pure endowment contract with single premium and loading $\varphi$ priced under the dynamic equivalence principle is more favorably priced than its counterpart with loading $\Psi$ and priced using a classical premium principle if the yearly share $\alpha \in(0,1]$ satisfies the following condition:

$$
\alpha \leq \frac{{ }_{t} p_{x}^{(0)}}{1-{ }_{t} p_{x}^{(0)}} \frac{\Psi-\varphi}{\pi_{0}^{(0)}+\varphi} .
$$

Proof. For a fixed benefit $b_{t}$, if $\Pi \leq P_{\Psi}$, then the dynamic contract is more favorably priced than the classical one. Since $P_{\Psi}$ is a constant, this inequality is always fulfilled if and only if the upper bound of the support of $\Pi$, that is

$$
P_{0}^{(0)}+\alpha \frac{P_{0}^{(0)}}{{ }_{t} p_{x}^{(0)}}\left(1-{ }_{t} p_{x}^{(0)}\right)
$$

is smaller than $P_{\Psi}$, which completes the proof.

\subsection{A viable risk sharing scheme}

It appears from Theorems 1 and 2 that an appropriate updating mechanism has to comply with two conditions. The first one aims at improving the solvency situation of the insurer whereas the second one follows from the fact that the contract has to remain appealing to policyholders. It is however important that both conditions do not conflict. In the following, we introduce a definition for a viable risk sharing scheme.

Definition 2 (Viable risk sharing scheme) Consider a pure endowment contract priced under a dynamic equivalence principle (characterized by a loading $\varphi$ and a yearly share $\alpha$ ) and a classical pure endowment (with loading $\Psi$ ). Moreover, assume that

$$
b_{t} v^{t} \leq P_{\Psi}
$$


holds in case:

$$
\Psi-\varphi \leq b_{t} v^{t}\left(1-{ }_{t} p_{x}^{(0)}\right) .
$$

Then, compared to the classical contract, the proposed updating mechanism improves the solvency situation of the insurer and is more favorably priced for policyholders if the following condition holds:

$$
\frac{\pi_{0}^{(0)}}{\Psi} \frac{\Psi-\varphi}{\pi_{0}^{(0)}+\varphi} \leq \alpha \leq \min \left\{\frac{{ }_{t} p_{x}^{(0)}}{1-{ }_{t} p_{x}^{(0)}} \frac{\Psi-\varphi}{\pi_{0}^{(0)}+\varphi}, \frac{\pi_{0}^{(0)}}{\pi_{0}^{(0)}+\varphi}\right\} .
$$

The above definition provides a condition on the contract such that both the insurer and the policyholders are better off with the updating mechanism. We can extract from (43) some limiting cases. On the one hand, setting $\varphi=\Psi$ leads to $\alpha=0$, which means that if the dynamic contract is as expensive as the classical one, then there must be no additional premiums. On the other hand, setting $\varphi=0$ leads to $\alpha=1$, which means that if the dynamic contract does not include any loading, then the policyholder should bear all the deviation risk. Moreover, imposing $\alpha \in[0,1]$ implies that the initial price of the dynamic contract has to be cheaper than that of the classical one, i.e. $\varphi \leq \Psi$. In this sense, the viable risk sharing scheme is consistent with intuition.

Inequality (41) ensures the existence of a range of $\alpha$ on which both parties agree in case (42) is fulfilled. Inequality (42) compares the difference between worst-estimate premium (i.e. when the $t$-year survival probability is set to 1 ) and the best-estimate premium (i.e. when the $t$-year survival probability is given by $\left.{ }_{t} p_{x}^{(0)}\right)$ with the difference between the loadings $\Psi$ and $\varphi$. Thus, these inequalities mean that if the difference between the prices of the classical and dynamic contracts is lower than the difference between the worst- and best-estimate premiums, then the loaded premium for the classical contract has to be at least equal to the worst-estimate premium.

\subsection{Analysis of the viable risk sharing scheme}

We study the pairs $(\alpha, \varphi)$ leading to a viable risk sharing scheme by considering three specific cases. The analysis is performed by determining the possible values of that pair for three different values of the ratio:

$$
\gamma=\frac{\Psi}{\pi_{0}^{(0)}} .
$$

For illustrative purpose only, assume that the time- 0 estimate of the $t$-year survival probability ${ }_{t} p_{x}^{(0)}$ is given by 0.981 . This number has been estimated from a Lee-Carter model fitted to the Belgian population and corresponds to that of a 30-year survival probability of a life aged 35. The estimation procedure follows the methodology described in Pitacco et al. (2009).

Figure 1 displays an example where $\gamma$ is such that inequality (41) is not satisfied. It is then straightforward to show that for $\varphi \geq 0$, inequality (42) will always be satisfied. In 


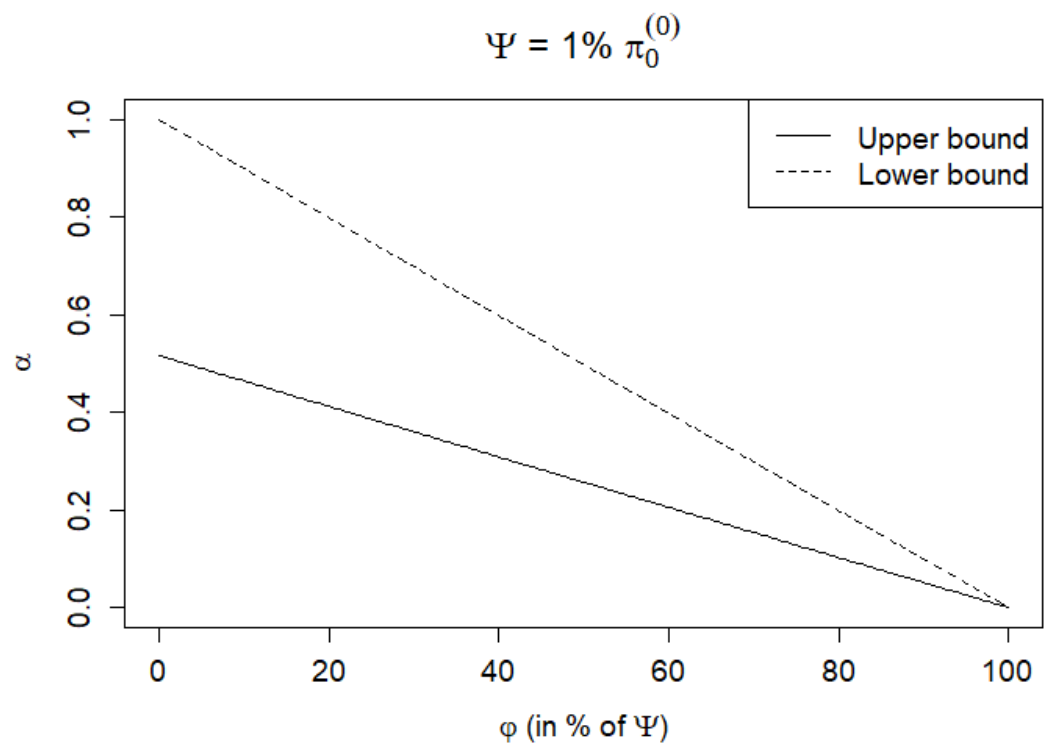

Figure 1: Bounds of $\alpha$ from inequality (43) as a function of $\varphi$, where $\gamma$ is such that inequality (42) is satisfied, while (41) is not.

this case, the lower bound from 43 is greater than the upper bound. As a consequence, we cannot find any $\alpha$ on which the insurer and policyholders would both agree. The reason is that the classical contract is too cheap. Thus, against a too cheap contract, policyholders would chose the dynamic one only if $\alpha$ is sufficiently low, and in particular, lower than the minimum required by the insurer to enhance its solvency situation. This translates into a conflict between the constraints of the two parties.

In the right panel of Figure 2, the ratio $\gamma$ is such that 41 becomes an equality. Again, we have that for $\varphi \geq 0$, inequality (42) is always fulfilled. Moreover, since (41) is an equality, we have that:

$$
\gamma=\gamma^{\star}=\frac{1-{ }_{t} p_{x}^{(0)}}{{ }_{t} p_{x}^{(0)}},
$$

and in this example we have $\gamma^{\star} \approx 1.94 \%$. Additionally, combining the fact that 42 is fulfilled with $\gamma=\gamma^{\star}$, we find that this case implies an equality between the upper and lower bounds from (43). This means that for each value of $\varphi$, there exists a unique value of $\alpha$ on which the two parties agree. In particular, any pair $(\alpha, \varphi)$ on that line of the graph will have the same loss probability for the insurer, and the same price for policyholders, taking into account their potential future payments. We can also conclude that a loading $\Psi=\gamma^{\star} \pi_{0}^{(0)}$ is the optimal loading for a classical contract in the sense of (43). The left panel of Figure 2 displays the ratio $\gamma^{\star}$ as a function of the initial estimate ${ }_{t} p_{x}^{(0)}$. Clearly, higher values of this estimate imply lower values of $\gamma^{\star}$. This would typically be the case for (relatively) short-term contracts or for young policyholders. Therefore, for high value of ${ }_{t} p_{x}^{(0)}$, the threshold level of the loading $\Psi$ (relatively to the pure premium) such that a viable risk sharing scheme can be determined will be very low.

In Figure 3, inequality 42 does not hold before the point $\varphi \approx 35.4 \% \Psi$, but it does 

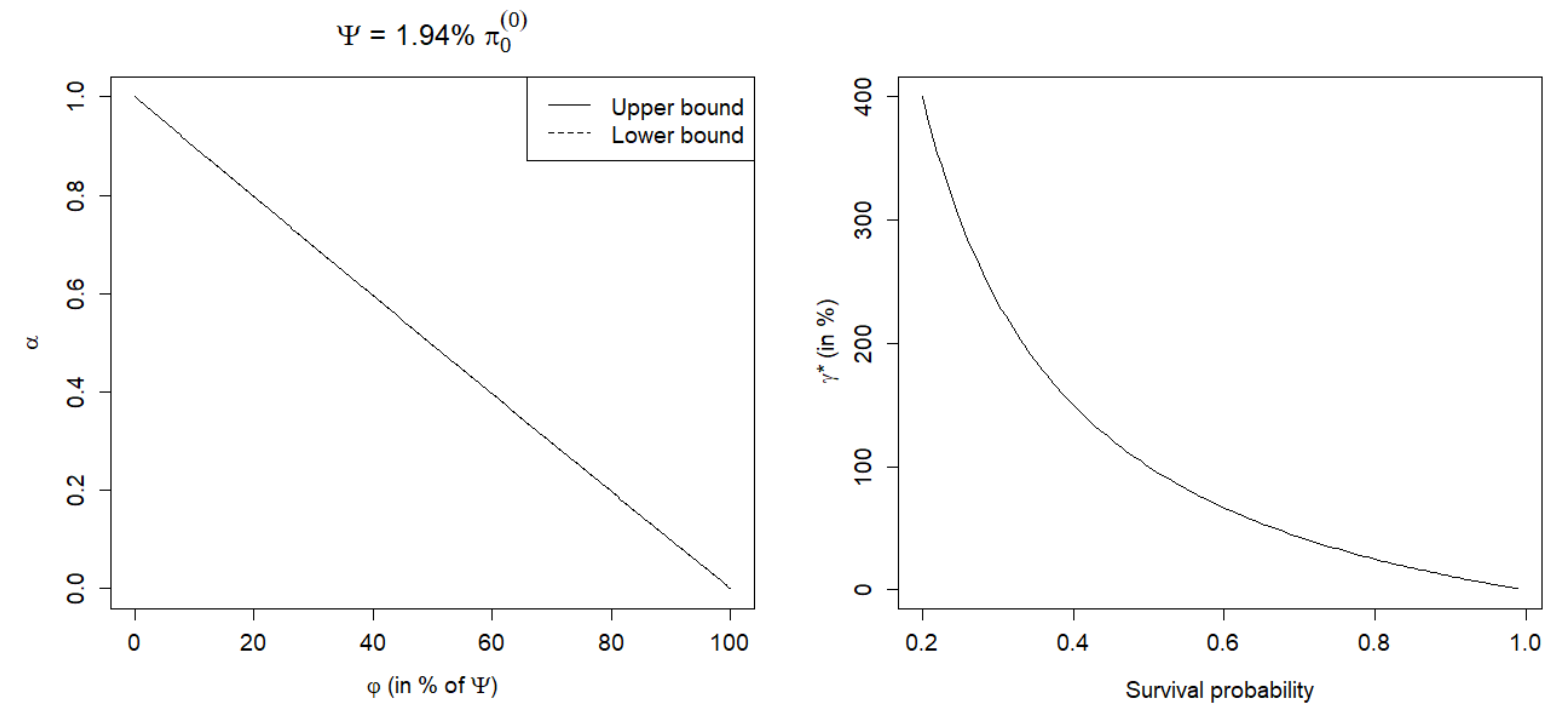

Figure 2: Right: Bounds of $\alpha$ from inequality (43) as a function of $\varphi$ where $\gamma$ is such that (41) becomes an equality. Left: Ratio $\gamma^{\star}$ as a function of the initial estimate of the $t$-year survival probability.

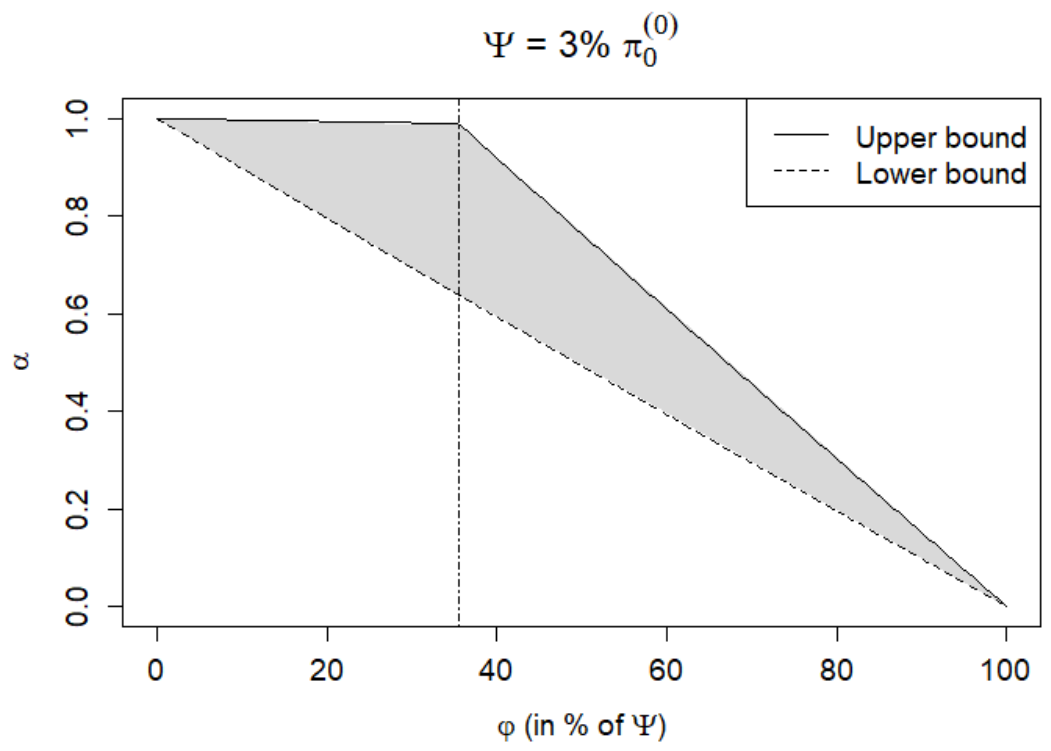

Figure 3: Bounds of $\alpha$ from inequality (43) as a function of $\varphi$, where $\gamma$ is such that inequality (41) is satisfied. The shaded area provides the possible $(\alpha, \varphi)$ leading to a viable risk sharing scheme. 
hold after that point. Moreover, the ratio $\gamma$ is chosen such that (41) is satisfied in both cases, although this condition is not necessary for the existence of $\alpha$ before $\varphi \approx 35.5 \% \Psi$. This implies that for each value of $\varphi$, we can find a range of $\alpha$ on which both parties agree. Thus, the possible pairs $(\alpha, \varphi)$ leading to a viable risk sharing scheme constitute a surface, which depends on the value of $\gamma$. In particular, we observe that if the loading of the classical contract is too high, then even for high values of $\varphi$ (i.e. before $\varphi \approx 35.4 \% \Psi$ ), policyholders would still prefer to bear a significant part of the deviation risk. Note that the point $\varphi \approx 35.4 \% \Psi$ is such that 42 becomes an equality, and thus, the ratio $35.4 \%$ from this example is determined from:

$$
1-\frac{\gamma^{\star}}{\gamma}
$$

\section{Concluding remarks}

In this paper, we have addressed systematic risk in long-term insurance business in a setting where both the assumption of independence and the assumption of known survival probabilities are violated. Increasing the size of the portfolio remains efficient for reducing the diversifiable part of the risk, but the deviation risk cannot be eliminated in this way. It appears that transferring the risk, or at least part of it, to policyholders is an efficient solution. However, in order for a risk sharing scheme to be viable, it should meet both the insurer's and the policyholders' constraints.

Any updating mechanism may suffer from a transparency drawback when the initial estimates are compared to the portfolio survival index. A solution coping with this issue consists in comparing the initial predictions with their corresponding realizations in a reference group (e.g. the general population of the country) instead of the realizations in the portfolio. This would enhance the transparency of the updating scheme, but in turn leaves the insurance company with an extra basis risk, on top of the random variations in the number of survivors. This approach can be a topic for future research.

Acknowledgments: The authors are grateful for the comments of two anonymous referees and for those of participants of the IME congress 2017 in Vienna, the CICIRM 2017 conference in Guilin and the Actuarial and Financial Mathematics conference 2018 in Brussels. The authors acknowledge the financial support of AG Insurance in the framework of the $A G$ Insurance Chair in Health Insurance at KU Leuven.

\section{References}

Blake, D., Cairns, A., Coughlan, G., Dowd, K. and MacMinn, R. (2013), 'The new life market', Journal of Risk and Insurance 80, 501-557.

Blake, D., El Karoui, N., Loisel, S. and MacMinn, R. (2017), 'Longevity risk and capital markets: The 2015-16 update', Insurance: Mathematics and Economics . 
Boon, L.-N., Briere, M. and Werker, B. (2018), 'Longevity risk: To bear or to insure?', https://papers.ssrn.com/sol3/papers.cfm?abstractid=2926902.

Chen, A., Hieber, P. and Klein, J. (2017), 'Tonuity: A novel individual-oriented retirement plan', Available at SSRN: https://ssrn.com/abstract $=3043013$.

Cox, S. and Lin, Y. (2007), 'Natural hedging of life and annuity mortality risks', North American Actuarial Journal 11, 1-15.

Dahl, M. (2004), 'Stochastic mortality in life insurance: market reserves and mortalitylinked insurance contracts', Insurance: Mathematics and Economics 35, 113-136.

Denuit, M., Dhaene, J., Goovaerts, M. and Kaas, R. (2005), Actuarial Theory for Dependent Risks: Measures, Orders and Models, Wiley.

Denuit, M., Dhaene, J., Hanbali, H., Lucas, N. and Trufin, J. (2017), 'Updating mechanism for lifelong insurance contracts subject to medical inflation', European Actuarial Journal 7, 133-163.

Denuit, M., Haberman, S. and Renshaw, A. (2011), 'Longevity-indexed life annuities', North American Actuarial Journal 15, 97-111.

Dhaene, J., Godecharle, E., Antonio, K., Denuit, M. and Hanbali, H. (2017), 'Lifelong health insurance covers with surrender values: updating mechanisms in the presence of medical inflation', ASTIN Bulletin 47, 803-836.

Feng, R. and Shimizu, Y. (2016), 'Applications of central limit theorems for equity-linked insurance', Insurance: Mathematics and Economics 69, 138-148.

Forman, J. B. and Sabin, M. J. (2016), 'Survivor funds', Pace Law Review 37.

Kaas, R., Goovaerts, M., Dhaene, J. and Denuit, M. (2008), Model actuarial risk theory using R, Springer.

Luciano, E., Regis, L. and Vigna, E. (2017), 'Single- and cross-generation natural hedging of longevity and financial risk', The Journal of Risk and Insurance (84), 961-986.

Majrek, D., Nowak, W. and Ziba, W. L. (2005), 'Conditional strong law of large number', International Journal of Pure and Applied Mathematics 20, 143-157.

Mauer, R., Mitchell, O. S., Rogalla, R. and Kartashov, V. (2013), 'Lifecycle portfolio choice with systematic longevity risk and variable investment-linked deferred annuities', The Journal of Risk and Insurance (80), 649-676.

Milevsky, M., Promislow, S. and Young, V. (2006), 'Killing the Law of Large Numbers: mortality risk premiums and the Sharpe ratio', Journal of Risk and Insurance 73, 673686.

Milevsky, M. and Salisbury, T. (2016), 'Equitable retirement income tontines: mixing cohorts without discriminating', ASTIN Bulletin: The Journal of the International Actuarial Association 46, 571-604. 
Piggott, J., Valdez, E. and Detzel, B. (2005), 'The simple analytics of a pooled annuity fund', Journal of Risk and Insurance 72, 497-520.

Pitacco, E., Denuit, M., Haberman, S. and Olivieri, A. (2009), Modelling Longevity Dynamics for Pensions and Annuity Business, Oxford University Press.

Richter, A. and Frederik, W. (2011), 'Mortality-indexed annuities: Managing longevity risk via product design', North American Actuarial Journal 15, 212-236.

Valdez, E., Piggott, J. and Wang, L. (2006), 'Demand and adverse selection in a pooled annuity fund', Insurance: Mathematics and Economics 39, 251-266.

Weale, M. and van de Ven, J. (2016), 'Variable annuities and aggregate mortalityrisk', National Institute Economic Review 237, 5561.

\section{A Appendix}

Building on the reasoning of Section 2, we have for $i \neq j$ that

$\mathbb{P}\left[I_{j}(0, t)=1 \mid I_{i}(0, t)=1\right]=\frac{\mathbb{E}\left[I_{i}(0, t) \times I_{j}(0, t)\right]}{\mathbb{E}\left[I_{i}(0, t)\right]}=\mathbb{P}\left[I_{j}(0, t)=1\right]+\frac{\operatorname{Cov}\left[I_{i}(0, t), I_{j}(0, t)\right]}{\mathbb{E}\left[I_{i}(0, t)\right]}$

Taking into account (6), we find that the $t$-year conditional survival probability of policyholder $j$, given that policyholder $i$ is alive at that time, can be expressed as

$$
\mathbb{P}\left[I_{j}(0, t)=1 \mid I_{i}(0, t)=1\right]=\mathbb{P}\left[I_{j}(0, t)=1\right]+\frac{\operatorname{Var}\left[{ }_{t} p_{x}(\Theta)\right]}{\mathbb{E}\left[{ }_{t} p_{x}(\Theta)\right]} \geq \mathbb{P}\left[I_{j}(0, t)=1\right] .
$$

From this expression, we see that the knowledge that policyholder $i$ survives increases the probability that policyholder $j$ survives. Additionally, the more uncertain ${ }_{t} p_{x}(\Theta)$, the more the conditional survival probability of $j$ exceeds the unconditional one.

The dependence between remaining lifetimes implies that the strong LLN is not applicable. In other words, increasing the portfolio size will not fully diversify the longevity risk. The classical strong LLN has to be replaced by the conditional strong LLN which states that

$$
\lim _{l_{x \rightarrow \infty}} \frac{1}{l_{x}} \sum_{i=1}^{l_{x}} I_{i}(0, t)=\mathbb{E}\left[I_{1}(0, t) \mid \Theta\right]={ }_{t} p_{x}(\Theta),
$$

almost surely; see e.g. Majrek et al. (2005). The limiting survivor proportion, or equivalently the average benefit, is a random variable which is related to the systematic part of the portfolio's risk per pure endowment policy.

However, it appears from Inequality (8) that by using the variance as a risk measure, increasing the size of the portfolio remains beneficial for the insurer. The benefit of 
diversification can also be illustrated via the tail value-at-risk which we denote for the random variable $X$ and the level $\epsilon \in(0,1)$ by $\operatorname{TVaR}_{\epsilon}[X]$. Hence,

$$
\operatorname{TVaR}_{\epsilon}[X]=\frac{1}{1-\epsilon} \int_{\epsilon}^{1} \operatorname{VaR}_{q}[X] \mathrm{d} q,
$$

where $\operatorname{VaR}_{q}[X]$ is the value-at-risk at the level $q \in(0,1)$.

The inequality

$$
\frac{1}{l_{x}+1} \operatorname{TVaR}_{\epsilon}\left[\sum_{i=1}^{l_{x}+1} I_{i}(0, t)\right] \leq \frac{1}{l_{x}} \operatorname{TVaR}_{\varepsilon}\left[\sum_{i=1}^{l_{x}} I_{i}(0, t)\right]
$$

holds for all probability levels $\epsilon$, and for any possible conditional dependence between the $I_{i}(0, t)$ 's; see e.g. Denuit et al. (2005) and Feng and Shimizu (2016). Thus, the relative contribution of each endowment policy to the solvency capital (determined as the TVaR minus the best estimate) decreases as the size of the portfolio increases. 